\title{
Crescimento econômico e habilidades cognitivas nos estados brasileiros
}

\begin{abstract}
Resumo
A importância do capital humano sobre o crescimento econômico serve de base para uma extensa literatura teórica e empírica em economia. No entanto, do ponto de vista empírico, não existe consenso sobre os melhores indicadores do estoque de capital humano. $\mathrm{O}$ presente trabalho busca observar essa relação através das habilidades cognitivas dos habitantes dos estados brasileiros. Para isso, utiliza-se como regressores, numa estimação de dados em painel com efeitos fixos, o desempenho dos alunos da quarta série do ensino fundamental e do terceiro ano do ensino médio na prova do Saeb (Sistema de Avaliação da Educação Básica) entre 1995-2005, em conjunto com uma variável tradicionalmente utilizada, os anos de estudo médios para pessoas com 25 anos ou mais. Os resultados mostram que, principalmente para o desempenho do terceiro ano e para os anos de estudo médios, existe relação entre o estoque de capital humano nos estados brasileiros e o crescimento de seu PIB per capita.
\end{abstract}

Palavras-chave: Crescimento econômico; Capital humano; Habilidades cognitivas

\begin{abstract}
The importance of human capital on economic growth is the basis for an extensive theoretical and empirical literature in economics. However, from an empirical point of view, there is no consensus on the best indicators of the stock of human capital. This study aims to observe the relationship through the cognitive skills of the inhabitants of the brazilian states. For this, it is used as regressors in a panel with fixed effects, the performance of students of the fourth grade students and third year of high school in the proof of Saeb (System of Basic Education Evaluation) between 1995-2005, together with a variable traditionally used, the average years of study for people with 25 years or more. The results show that, especially for the third year and the average years of study, there is a relationship between the stock of human capital in the brazilian states and the growth of GDP per capita.
\end{abstract}

Keywords: Economic growth, human capital, cognitive skills. 


\section{INTRODUÇÃO}

Durante a década de 1980, os chamados modelos de crescimento endógeno, como de Romer (1986, 1987, 1990a), Lucas (1988) e Becker, Murphy e Tamura (1990) trouxeram novamente à tona a importância do capital humano sobre o crescimento econômico, resgatando as ideias pioneiras de Arrow (1962), Nelson e Phelps (1966) e Uzawa (1964). Durante o início da década de 1990 surgiu uma série de avaliações empíricas que buscavam interpretar a relação entre capital humano e crescimento, utilizando em especial aspectos educacionais como indicador do capital humano acumulado em cada país.

Entre os trabalhos, vale destacar Romer (1990b), que utiliza variáveis como taxa de alfabetização e investimentos governamentais para estimar o capital humano, Barro (1991), Levine e Renelt (1992) e Mankiw, Romer e Weil (1992), que utilizam a taxa de matrículas e anos de estudo médios, Barro e Lee (1993) que ,por sua vez, buscam melhorar as proxies de capital humano ao incluir como variáveis a partir de sete níveis educacionais, por exemplo, pessoas com primário e secundário completos ou incompletos. Benhabib e Spiegel (1994), atentam para as diferenças do impacto indireto do capital humano o crescimento via inovações tecnológicas e do impacto direto via aumento da produtividade da mão de obra.

Hanushek e Kimko (2000) e Lee e Barro (2001) percebem falhas nas variáveis comumente utilizadas para indicar o capital humano, como, por exemplo, o fato de que ao considerar os anos de estudo médios, o retorno de cada ano em cada país seria igual, ou seja, desconsidera-se a diferença de qualidade de ensino em cada país. Como ressalta Hanushek e Woessman (2012a) o capital humano não é diretamente observável, e um indicador confiável são as habilidades cognitivas dos indivíduos, aos moldes do proposto por Cunha et al (2006), e observadas via testes padrão internacionais de matemática e ciências por exemplo.

As vantagens apresentadas por Hanushek e Woessman (2012a) destacam que as habilidades cognitivas englobam dimensões do capital humano mais amplas que as apenas observadas via indicadores escolares, essas vantagens seriam (i), o desempenho nos testes de conhecimento englobam aspectos que a escola se esforça para produzir, (ii), os resultados não irão apenas incorporar o conhecimento adquirido na escola, mas também aspectos familiares, que como observa Cunha et al (2006) são fatores importantes para as habilidades cognitivas e não cognitivas, e também incorpora as diferenças na 
própria habilidade não adquirida e (iii), as diferenças em níveis escolares possibilita observações úteis as políticas educacionais posteriores. Assim sendo, uma série de trabalhos aborda o capital humano via habilidades cognitivas, em particular buscando um indicador único para testes padrão de conhecimento em áreas como matemática, ciências e leitura, como por exemplo, em Hanushek e Kimko (2000), Bils e Klenow (2000), Lee e Barro (2001), Hanushek e Woessman (2008, 2011, 2012b).

Dessa forma, a partir da abordagem das habilidades cognitivas, o presente trabalho busca observar a relação entre capital humano e crescimento econômico nos estados brasileiros. Para isso, utiliza-se uma regressão com dados em painel com os resultados da prova do Saeb (Sistema de Avaliação da Educação Básica) de matemática para os alunos da quarta série do ensino fundamental e para o terceiro ano do ensino médio, entre 1995 e 2005, sendo a prova bianual, como indicadores das habilidades cognitivas em cada estado. Para isso buscou-se um indicador padronizado com possibilidade de utilização para todos os estados em todos os anos cobertos pela amostra. A decisão pelo período de 1995-2005 deve-se ao fato da prova do Saeb ter sido interrompida em 2007 e 2009, anos em que foi realizada a Prova Brasil.

Assim sendo, a Seção 2 apresenta um modelo de formação de capital humano via habilidades, como exposto em Cunha e Heckman (2007), e a forma como as habilidades se inserem numa função produção e geram crescimento econômico posterior, como visto em Hanushek e Woessman (2012a). A Seção 3 descreve as variáveis utilizadas e os resultados das estimações para os estados brasileiros. Por fim, a Seção 4 apresenta as considerações finais e conclusões a partir dos resultados encontrados nas seções anteriores.

\section{CRESCIMENTO ECONOMICO E CAPITAL HUMANO}

A presente seção busca de maneira sucinta apresentar as relações entre crescimento econômico e capital humano. Para isso, inicialmente apresenta-se a tecnologia de formação do capital humano, como proposta por Cunha et al (2006) e Cunha e Heckman (2007), bem como diversos resultados encontrados na literatura a respeito da formação do capital humano com ênfase nas habilidades cognitivas e não cognitivas. Na sequência é observado um modelo simples de crescimento com capital humano, aos moldes do apresentado por Hanushek e Woessman (2012a). 


\subsection{HABILIDADES COGNITIVAS, NÃO COGNITIVAS E CAPITAL HUMANO}

Cunha e Heckman (2007) e Cunha et al (2006), apresentam a tecnologia da formação das habilidades cognitivas e não cognitivas, que resultam no capital humano, desenvolvendo um modelo com base em resultados empíricos previamente existentes na literatura. Cunha e Heckman (2007) destacam três observações bem estabelecidas sobre a importância das habilidades: primeiro, as habilidades cognitivas são um previsor do sucesso socioeconômico (Heckman (1995)), segundo, as habilidades não cognitivas, como autocontrole e disciplina, por exemplo, possuem impacto direto sobre a produtividade do indivíduo (Heckman, Stixrud e Urzua (2006) e Urzua (2006)), e terceiro, a distinção entre natureza e educação (nature versus nurture) é obsoleta, já que as habilidades são produzidas e mesmo as características genéticas são suscetíveis a influência do ambiente (Rutter (2006)).

Estabelecidas as três observações iniciais, Cunha e Heckman desenvolvem um modelo a partir de seis resultados da literatura empírica, sendo eles (i) as lacunas de habilidade entre indivíduos e grupos sociais, tanto no aspecto cognitivo como não cognitivo iniciam nos primeiros anos de vida, como visto em Carneiro e Heckman (2003), Cunha et al (2006) e Blau e Currie (2006), (ii) existem períodos críticos e sensíveis na formação das habilidades, sendo o conceito de período sensível aquele em que as habilidades são adquiridas com maior eficiência dentro de um intervalo de tempo na vida do indivíduo, enquanto o período crítico é aquele em que certas habilidades são desenvolvidas apenas naquele período, Knusdsen (2004), (iii) embora a literatura apresente baixo retorno em intervenções em adolescentes, intervenções em crianças desfavorecidas apresentam alto retorno, Barnett (2004), Cunha et al (2006). Como apresentam Shonkoff e Phillips (2000), diferentes habilidades são formadas em diferentes períodos da vida.

Seguindo, (iv) se o investimento em crianças em situação desfavorável não for seguido por investimentos posteriores, seu efeito sobre os anos seguintes é perdido, o que reforça a complementaridade entre investimentos no ciclo de vida, Currie e Thomas (1995), Cunha et al (2006), (v) o efeito da restrição de crédito no resultado da criança quando chega a fase adulta, depende em que período da infância do indivíduo a possibilidade de maior investimento na educação existia, por exemplo, Carneiro e Heckman $(2002,2003)$ documentam que renda familiar maior durante os anos em que o 
indivíduo poderia estar na faculdade tem pouco efeito sobre a entrada dos mesmos na própria faculdade, e por fim (vi) habilidades não cognitivas potencializam as habilidades cognitivas e são importantes no sucesso das famílias e nos retornos em intervenções sobre famílias em desvantagem, Heckman Stixrud e Urzua (2006).

O modelo de Cunha e Heckman (2007) ressalta a formação das habilidades em múltiplos períodos da infância, diferente dos modelos tradicionais como o de Becker e Tomes (1986), por exemplo, em que toda a infância é tratada como um único período. É possível considerar um modelo de gerações sobrepostas, com os indivíduos vivendo dois períodos $2 T$, por exemplo, onde nos primeiros $T$ anos de vida o indivíduo é uma criança com pais adultos, e de $T+1$ até $2 T$ é adulto e pai de alguma criança. Os investimentos na educação das crianças são representados por $I_{t}$, com $t=1,2, \ldots, T$. O vetor de habilidades pode ser dividido em habilidades cognitivas e não cognitivas e apresentado como $\theta_{t}=\left(\theta_{t}^{C}, \theta_{t}^{N}\right)$, onde cada agente nasce com a condição inicial $\theta_{1}$. A evolução das habilidades no tempo, para uma criança com $t$ anos, pode ser expressa por:

$$
\theta_{t+1}=f_{t}\left(h, \theta_{t}, I_{t}\right)
$$

Onde $h$ representa as características dos pais, e $f_{t}$ é uma função estritamente crescente, estritamente côncava em $I_{t}$, e duas vezes diferenciável. Utilizando substituição recursiva, é possível apresentar $\theta_{t+1}$ como função de $\theta_{1}$ e dos investimentos ao longo da vida da criança $t=(1, \ldots, T)$ :

$$
\theta_{t+1}=m_{t}\left(h, \theta_{1}, I_{1}, \ldots, I_{t}\right)
$$

Dois aspectos importantes podem ser extraídos do modelo, a complementaridade dinâmica e a auto-produtividade. A complementaridade dinâmica ocorre quando o estoque de habilidades adquiridas em algum período torna o investimento naquele período mais produtivo, o que implica que estudantes com maiores habilidades cognitivas e não cognitivas iniciais são mais aptos a adquirir ambas posteriormente. Formalmente:

$$
\frac{\partial^{2} f_{t}\left(h, \theta_{t}, I_{t}\right)}{\partial \theta_{t}, I_{t}^{\prime}}>0
$$


Já a auto-produtividade significa que maiores estoques de habilidade em algum período criam maiores estoques de habilidade em períodos seguintes:

$$
\frac{\partial f_{t}\left(h, \theta_{t}, I_{t}\right)}{\partial \theta_{t}}>0
$$

Os efeitos conjuntos explicam o porquê de existirem altos retornos em investimentos em crianças em desvantagem e baixo retorno no investimento em adolescentes em desvantagem. Também é possível observar que para crianças em idades mais avançadas existe um trade-off entre eficiência e equidade em termos de investimento educacional, o que, no entanto, não ocorre para investimento em crianças mais jovens, na pré-escola, por exemplo. O período crítico, para $h, \theta_{1}, I_{1}, \ldots, I_{t}, s \neq t$, pode ser expresso como:

$$
\begin{aligned}
& \frac{\partial \theta_{t+1}}{\partial I_{s}}=\frac{\partial m_{t}\left(h, \theta_{1}, I_{1}, \ldots, I_{t}\right)}{\partial I_{s}}=0 \\
& \frac{\partial \theta_{t+1}}{\partial I_{t *}}=\frac{\partial m_{t}\left(h, \theta_{1}, I_{1}, \ldots, I_{t}\right)}{\partial I_{t *}}>0
\end{aligned}
$$

Ou seja, o investimento em determinada habilidade só irá gerar efeito sobre o estoque de habilidades do indivíduo se for realizado no período $t$ *. Já o período sensível, considerando os mesmos níveis de $h, \theta_{1}, I_{1}, \ldots, I_{t}$, pode ser visto em:

$$
\frac{\partial \theta_{t+1}}{\partial I_{s}}<\frac{\partial \theta_{t+1}}{\partial I_{t *}}
$$

Para o período ser sensível, investimentos no período $t *$ são mais produtivos do que investimentos realizados no período $S$, se em ambos os casos os insumos da função $\theta_{t+1}$ forem iguais. Cunha e Heckman (2007) apresentam três possibilidades para a função formadora das habilidades. Primeiro, os investimentos poderiam ser substitutos perfeitos, ou seja, não haveria diferença entre investir em cada fase do ciclo de vida do indivíduo em termos de resultado sobre as habilidades, o resultado dessa forma funcional se assemelha a função apresentada por Becker e Tomes (1986), por exemplo. Baixo investimento na infância poderia ser compensado com maiores investimentos na 
adolescência, por exemplo, o que contraria as evidências empíricas. Segundo, poderiam ser complementares perfeitos, nesse caso, seriam necessários investimentos em cada período do ciclo de vida, caso contrário o resultado em termos de ganho nas habilidades seria igual a zero. Embora a complementaridade seja observável em termos empíricos, como em Currie e Thomas (1995), o caso extremo de complementaridade perfeita não encontra respaldo empírico, já que como visto, embora apresentem retornos mais baixos que o investimento em crianças em desvantagem, o investimento em adolescentes em desvantagem ainda assim apresenta retornos positivos, Cunha et al (2006).

Cunha e Heckman (2007) apresentam uma forma funcional mais geral que as citadas, sendo uma CES. Considerando por simplicidade $T=2$ e $h^{\prime}=\theta_{3}$ como sendo as características dos pais, têm-se:

$$
h^{\prime}=m_{2}\left(h, \theta_{1},\left[\gamma\left(I_{1}\right)^{\phi}+(1-\gamma)\left(I_{2}\right)^{\phi}\right]^{1 / \phi}\right)
$$

Onde $\gamma$ é o multiplicador das habilidades, revelando como o investimento inicial afeta não só $h^{\prime}$, mas também a produtividade de $I_{2}$ sobre $\theta_{2}$. Já o parâmetro $\phi$ é a elasticidade de substituição entre os investimentos em idades mais jovens ou mais avançadas do indivíduo. Para $\phi$ baixo, um investimento inicial baixo não é facilmente compensado em investimentos posteriores na formação do capital humano.

Cunha et al (2006) apresentam a relação entre o investimento ótimo e a idade, a partir das evidências empíricas:

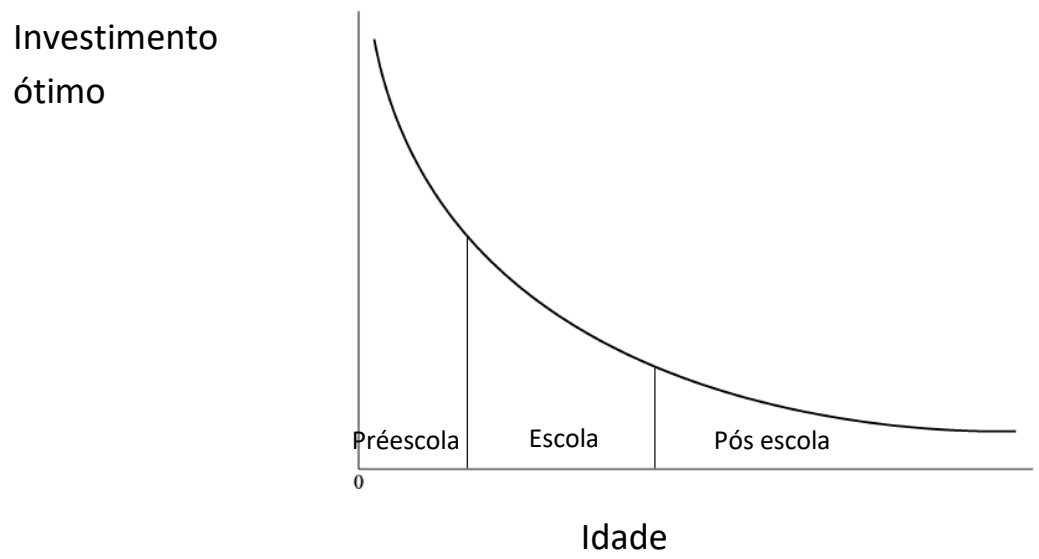

Figura 1 - Níveis ótimos de investimento no ciclo de vida.

Fonte: Cunha et al (2006) 
Outro aspecto relevante para a formação do capital humano são as restrições de crédito a nível escolar. Cunha e Heckman (2007) observam o retorno ótimo associado ao investimento em educação e sua relação com as restrições existentes. Já Carneiro e Heckman $(2002,2003)$ analisam o impacto da renda em diferentes períodos do ciclo de vida e como isso irá impactar no resultado em termos de habilidades e sucesso socioeconômico posterior.

\subsection{CRESCIMENTO E HABILIDADES COGNITIVAS}

Diversos modelos apresentam a relação entre crescimento e capital humano, entre eles é possível destacar Romer (1986, 1987 e 1990) com destaque para o papel da tecnologia sobre o crescimento e Lucas (1988), que insere o capital humano, aos moldes do proposto por Becker (1964), diretamente na função produção. A Abordagem aqui utilizada baseia-se em Hanushek e Woessman (2012a), partindo de um modelo simples diretamente ligado a estimação posterior e sem observar todas as implicações teóricas identificadas nos trabalhos anteriormente citados. A partir de uma especificação do tipo Cobb-Douglas, é possível inserir o capital humano diretamente na função produção, onde $y$ é o PIB per capita, $k$ o estoque de capital físico per capta e $h$ o estoque de capital humano per capita. Transformando em logaritmo e acrescentando um termo de erro têmse:

$$
\log y_{i t}=\alpha \log k_{i t}+\beta \log h_{i t}+\epsilon_{i t}
$$

Como exposto por Hanushek e Woessman (2012a), o capital humano pode ser apresentado por:

$$
H=\gamma F+\varphi(q S)+\delta A+\mu Z+u
$$

Onde $F$ representa os inputs provenientes da família, $q S$ a quantidade e qualidade dos inputs provenientes da escola, $A$ as habilidades individuais e $Z$ outros fatores como a experiência no trabalho e a saúde. Assim sendo, os testes de conhecimentos ao destacar as habilidades cognitivas acabam por abranger diversos elementos do capital humano, como as habilidades individuais e os inputs das famílias e escolas. Além disso, como destaca Cunha et al (2006), embora a ênfase dos trabalhos como Hanushek e Kimko 
(2000) e Hanushek e Woessman (2012a) esteja nas habilidades cognitivas observadas pelos testes, o bom desempenho é potencializado pelas habilidades não cognitivas, como paciência, autocontrole e disciplina, que geram maior produtividade nos estudos e consequentemente nos resultados cognitivos. Assim, parte dos resultados dos testes de matemática se deve a inter-relação entre habilidades cognitivas e não cognitivas.

\section{DADOS UTILIZADOS E RESULTADOS}

A presente seção apresenta os dados utilizados, sendo eles o PIB per capita, o PIB da indústria de transformação, também per capita, os resultados padronizados da prova do Saeb para os alunos da quarta série do ensino fundamental e para o terceiro ano do ensino médio e os anos médios de estudo para pessoas de 25 anos ou mais, nos estados brasileiros, entre 1995-2005. Também são realizadas nesta seção estimações com dados em painel, observando a relação entre habilidades cognitivas e crescimento econômico no período e regiões citadas.

\subsection{DADOS E MODELO ECONOMÉTRICO}

Os dados utilizados para o PIB per capita foram retirados do IPEADATA, sendo o PIB real a preços de 1995, deflacionado pelo IPCA. A proxy para o capital físico per capita foi o PIB real da indústria de transformação per capita, a preços de 2000 e também deflacionado pelo IPCA. Quanto aos indicadores de desempenho nas provas de matemática do Saeb, foram utilizados os microdados das provas, criando um índice em que os piores resultados individuais anuais assumiam valor zero e os melhores, valor cem. Assim todas as notas foram recalculadas como uma proporção das melhores notas, sendo que, por exemplo, a média do terceiro ano em Santa Catarina no ano de 2005, que foi de 52.92, significa que a média de desempenho dos estudantes de Santa Catarina foi de $52.92 \%$ da melhor nota individual observada no Brasil naquele ano. A Figura 2 mostra o desempenho médio da quarta série por estado e por ano, as barras apresentam o intervalo de confiança de $95 \%$ para as médias: 

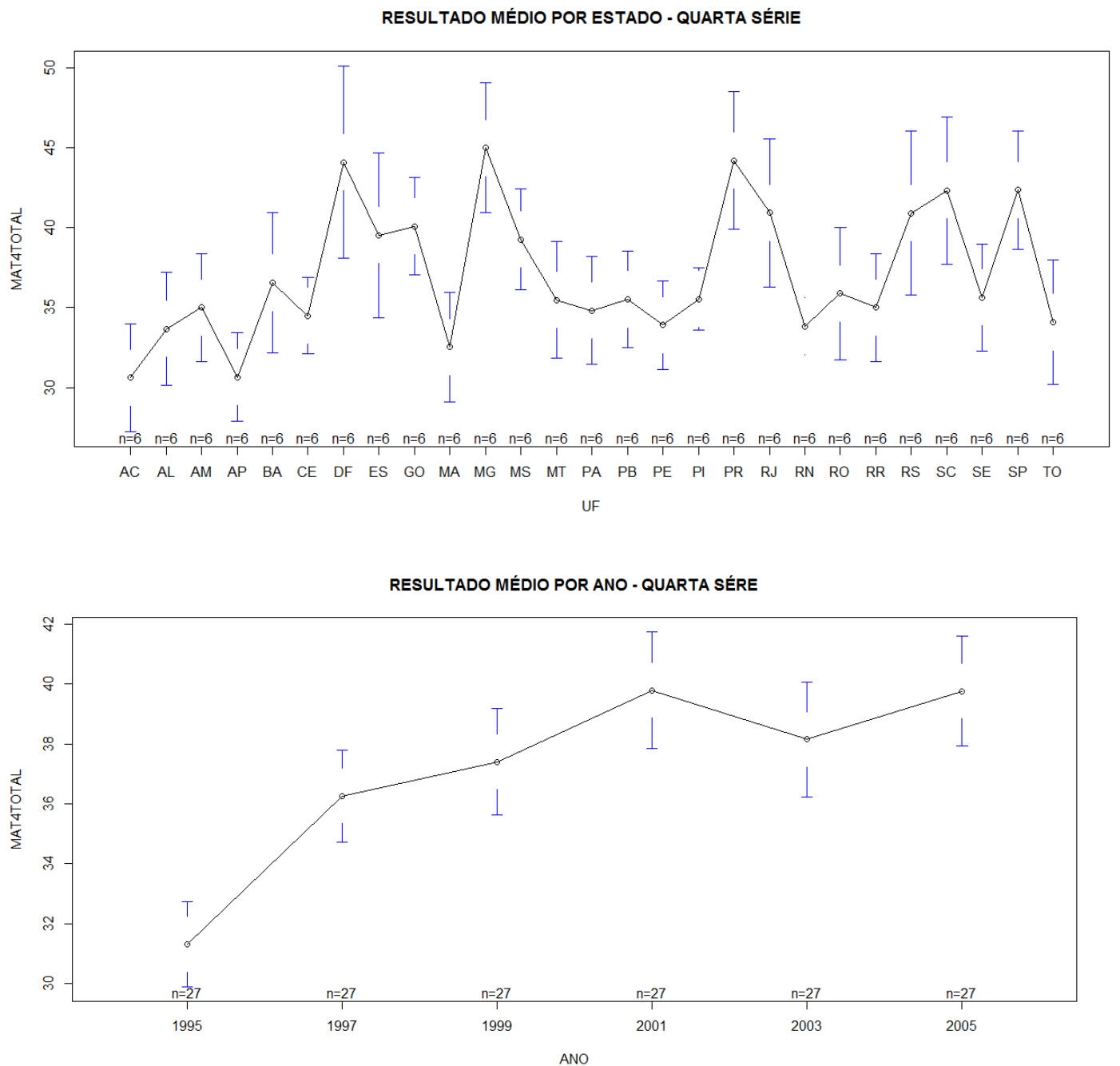

Figura 2 - Resultado médio das provas de matemática do Saeb da quarta série do ensino fundamental, por estado e por ano, de 1995-2005.

Fonte: Elaborado pelo autor (2016)

É possível observar crescimento nos resultados médios anuais, que estavam próximos a 31 em 1995 e chegaram próximos a 40 em 2005. Quanto ao desempenho dos estados no período, as melhores médias pertencem a Minas Gerais, Distrito Federal e Paraná, enquanto as piores pertencem a Acre, Amapá e Maranhão. A Figura 3 apresenta os mesmos resultados para o terceiro ano do ensino médio: 


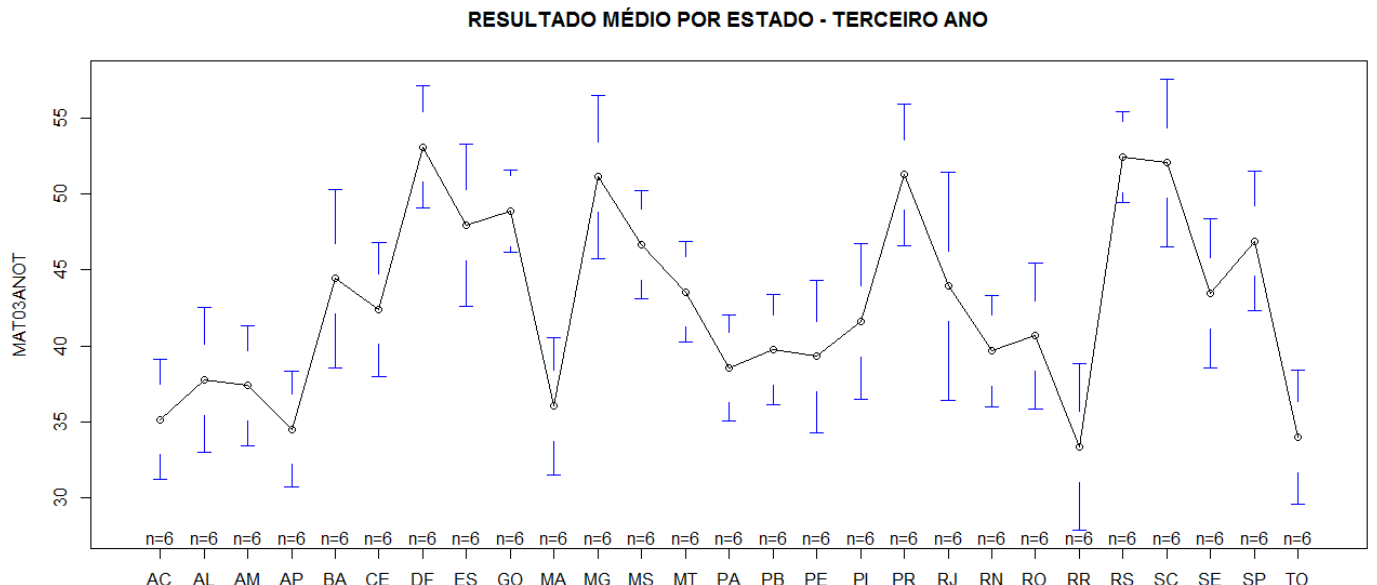

UF

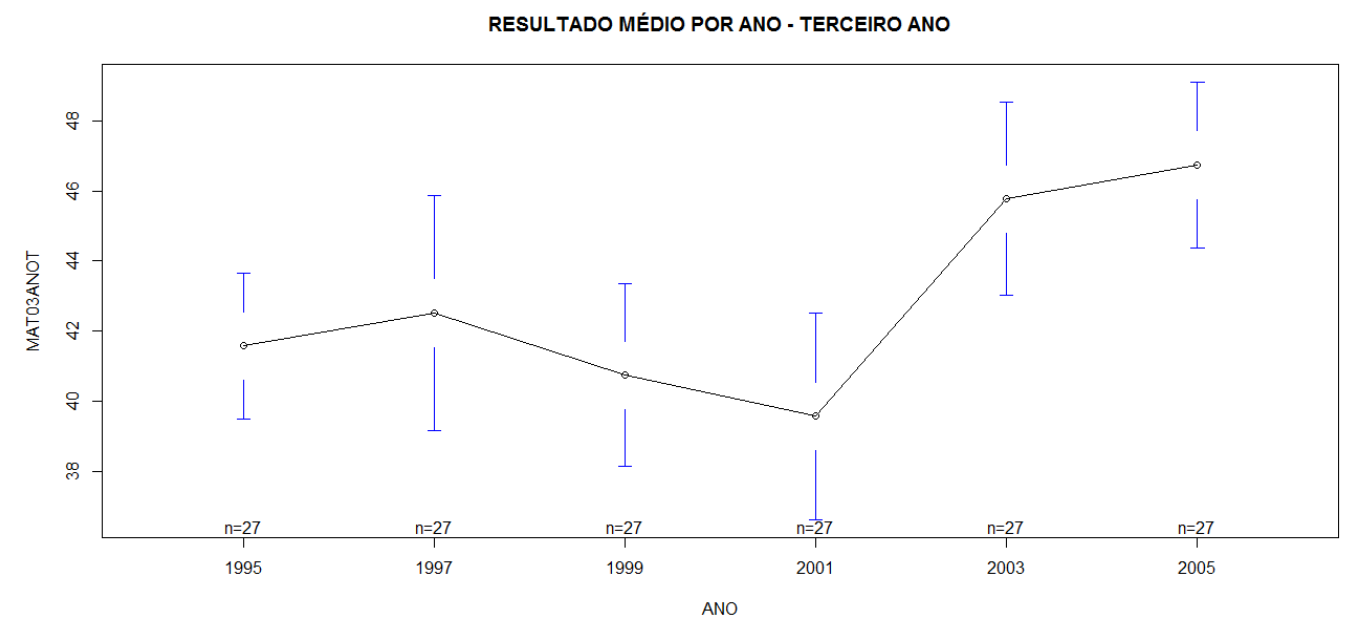

Figura 3 - Resultado médio das provas de matemática do Saeb do terceiro ano do ensino médio, por estado e por ano, de 1995-2005.

Fonte: Elaborado pelo autor (2016)

Nesse caso, houve queda no desempenho entre 1997 e 2001, e crescimento substancial entre 2001 e 2005. A série inicia-se com um valor próximo a 41, e se aproxima de 46 em seu final. Os estados com melhor desempenho foram o Distrito Federal, Santa Catarina e Rio Grande do Sul, enquanto os piores desempenhos foram observados em Roraima, Tocantins e Amapá. Quanto aos anos de estudo médios, extraídos da Pnad, podem ser observados na Figura 4: 

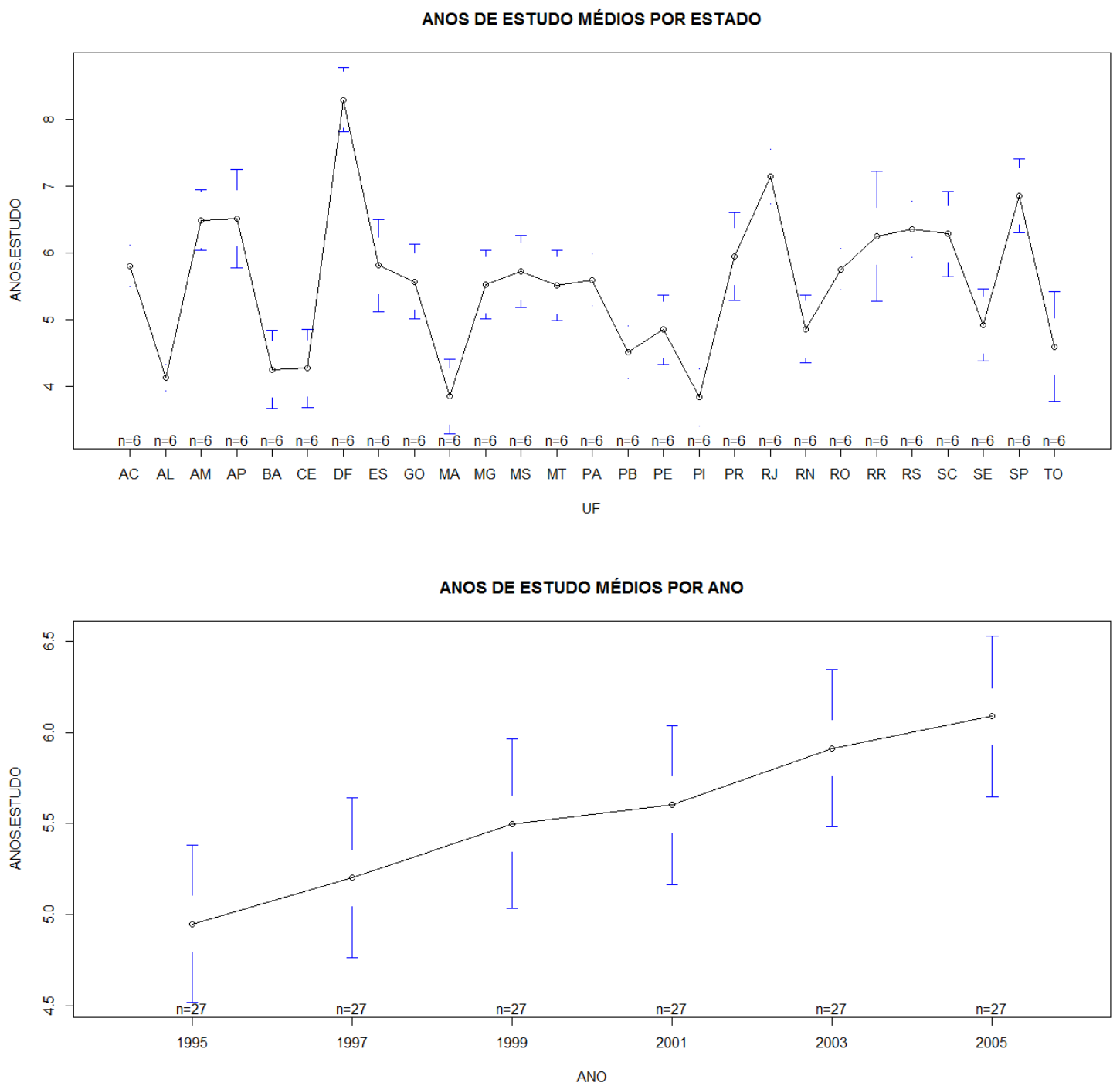

Figura 4 - Anos de estudo médios para pessoas de 25 anos ou mais, por estado e por ano, de 1995-2005.

Fonte: Elaborado pelo autor (2016)

Em relação aos anos de estudo médios, houve um crescimento médio contínuo entre 1995 e 2005. Já para os estados, as melhores médias no período pertencem a São Paulo, Rio de Janeiro e Distrito Federal, enquanto as piores médias são vistas no Piauí, Maranhão e Alagoas. Alguns indícios podem ser observados nos dados, como a existência de complementaridade nos resultados das provas entre quarta série e terceiro ano. Em geral, estados com melhor desempenho na quarta série apresentam melhor desempenho também no terceiro ano, o que está de acordo com a ideia proposta por Cunha e Heckman (2007) e com as observações de que o processo de aprendizado é cumulativo e com etapas distintas. Também é possível observar a princípio que as regiões com melhor desempenho 
nas provas e com maior média de anos de estudo também são as com maior renda média, sendo elas o Sul e Sudeste acrescidos do Distrito Federal.

Para as regressões foram utilizadas sete formas funcionais para o capital humano, combinando os resultados das provas da quarta série e do terceiro ano e os anos de estudo médios nos estados, como apresentado no Quadro 2. Além disso inclui-se a proxy para o capital físico em todas as regressões.

\subsection{RESULTADOS}

Ao utilizar dados em painel nas sete especificações apresentadas no Quadro 2, inicialmente é preciso observar a existência de heterogeneidade não observável nos resíduos, e caso ela exista, observar sua correlação com os regressores. Em caso de não existência, é possível utilizar um modelo simples de mínimos quadrados ordinários. Para observar a existência de heterogeneidade tanto em termos de cross-section, quanto de período foram utilizados os testes presentes no Quadro 1, sendo eles um teste F, e de Breusch-Pagan para os efeitos individuais dos estados e um teste de Breusch-Pagan para heterogeneidade nos períodos. Em todas as sete especificações observou-se efeitos individuais nos estados. Com relação aos períodos, em apenas duas regressões existe efeitos individuais, sendo a terceira especificação, em que os regressores são o PIB na indústria de transformação per capita e o resultado do terceiro ano no Saeb, e a quinta especificação, em que os regressores são o PIB da indústria de transformação per capita e o resultado do Saeb para a quarta série e para o terceiro ano. Quanto à escolha entre efeitos fixos e aleatórios, que depende da correlação do resíduo com os regressores, o Quadro 1 apresenta o teste de Hausman, que atesta a utilização de efeitos fixos na estimação.

A primeira estimação, presente no Quadro 2, que utiliza a proxy para o capital per capita e o resultado do Saeb para a quarta série, apresentou sinal positivo e resultado com significância estatística para o teste, o que implica que as habilidades cognitivas, quando observadas para alunos da quarta série, pesam para o crescimento do PIB. No entanto, ao acrescentar os anos de estudo médio para pessoas com vinte e cinco anos ou mais em cada estado, o que é realizado na especificação (2), o resultado da Saeb perde a significância estatística, enquanto os anos de estudo possuem coeficiente positivo e significante num intervalo de $99 \%$ de confiança. Dessa forma, há indícios de colinearidade entre as 


\begin{tabular}{|c|c|c|c|c|c|c|c|}
\hline TESTE & (1) & (2) & (3) & (4) & (5) & (6) & (7) \\
\hline $\begin{array}{l}\text { Teste F - heterogeneidade não } \\
\text { observável (individual) }\end{array}$ & $\begin{array}{l}F=269.26 \\
p=0.0000\end{array}$ & $\begin{array}{l}F=124.5 \\
p=0.0000\end{array}$ & $\begin{array}{l}F=292.11 \\
p=0.0000\end{array}$ & $\begin{array}{l}F=123.27 \\
p=0.0000\end{array}$ & $\begin{array}{l}F=300.96 \\
p=0.0000\end{array}$ & $\begin{array}{l}F=129.63 \\
p=0.0000\end{array}$ & $\begin{array}{l}F=123.81 \\
p=0.0000\end{array}$ \\
\hline $\begin{array}{l}\text { Teste de Breusch-Pagan - } \\
\text { heterogeneidade não } \\
\text { observável (individual) }\end{array}$ & $\begin{array}{c}\text { Chi-squared }=294.24 \\
p=0.0000\end{array}$ & $\begin{array}{c}\text { Chi-squared }=112.65 \\
p=0.0000\end{array}$ & $\begin{array}{c}\text { Chi-squared }=344.16 \\
p=0.0000\end{array}$ & $\begin{array}{c}\text { Chi-squared }=95.201 \\
p=0.0000\end{array}$ & $\begin{array}{c}\text { Chi-squared }=314.78 \\
p=0.0000\end{array}$ & $\begin{array}{c}\text { Chi-squared }=163.52 \\
p=0.0000\end{array}$ & $\begin{array}{c}\text { Chi-squared }=90.042 \\
p=0.0000\end{array}$ \\
\hline $\begin{array}{l}\text { Teste de Breusch-Pagan - } \\
\text { heterogeneidade não } \\
\text { observável (tempo) }\end{array}$ & $\begin{array}{c}\text { Chi-squared }=4.934 \\
p=0.0255\end{array}$ & $\begin{array}{c}\text { Chi-squared }=105.21 \\
p=0.0000\end{array}$ & $\begin{array}{c}\text { Chi-squared }=1.937 \\
p=0.164\end{array}$ & $\begin{array}{c}\text { Chi-squared }=94.63 \\
p=0.0000\end{array}$ & $\begin{array}{c}\text { Chi-squared }=0.4961 \\
p=0.4812\end{array}$ & $\begin{array}{c}\text { Chi-squared }=24.348 \\
p=0.0000\end{array}$ & $\begin{array}{c}\text { Chi-squared }=113.3 \\
p=0.0000\end{array}$ \\
\hline $\begin{array}{l}\text { Teste de Hausman - efeitos } \\
\text { fixos ou aleatórios }\end{array}$ & $\begin{array}{c}\text { Chi-squared }=24.62 \\
p=0.0000\end{array}$ & $\begin{array}{c}\text { Chi-squared }=46.49 \\
\qquad p=0.0000\end{array}$ & $\begin{array}{c}\text { Chi-squared }=44.796 \\
p=0.0000 \\
\end{array}$ & $\begin{array}{c}\text { Chi-squared }=40.788 \\
p=0.0000\end{array}$ & $\begin{array}{c}\text { Chi-squared }=49.374 \\
p=0.0000\end{array}$ & $\begin{array}{c}\text { Chi-squared }=32.296 \\
p=0.0000\end{array}$ & $\begin{array}{c}\text { Chi-squared }=41.967 \\
\quad p=0.0000\end{array}$ \\
\hline TESTE & (1) & (2) & (3) & (4) & (5) & (6) & (7) \\
\hline $\begin{array}{l}\text { Teste de Breusch-Pagan - } \\
\text { Correlação na cross-section }\end{array}$ & $\begin{array}{c}\text { Chi-squared }=758.96 \\
p=0.0000\end{array}$ & $\begin{array}{c}\text { Chi-squared }=642.67 \\
p=0.0000\end{array}$ & $\begin{array}{c}\text { Chi-squared }=808.14 \\
p=0.0000\end{array}$ & $\begin{array}{c}\text { Chi-squared }=606.52 \\
p=0.0000\end{array}$ & $\begin{array}{c}\text { Chi-squared }=621.7 \\
p=0.0000\end{array}$ & $\begin{array}{c}\text { Chi-squared }=614.69 \\
p=0.0000\end{array}$ & $\begin{array}{c}\text { Chi-squared }=628.73 \\
p=0.0000\end{array}$ \\
\hline $\begin{array}{l}\text { Teste de Pesaran - Correlação } \\
\text { na cross-section }\end{array}$ & $\begin{array}{l}z=17.671 \\
p=0.0000\end{array}$ & $\begin{array}{l}z=12.342 \\
p=0.0000\end{array}$ & $\begin{array}{l}z=12.731 \\
p=0.0000\end{array}$ & $\begin{array}{l}z=5.1833 \\
p=0.0000\end{array}$ & $\begin{array}{c}z=7.033 \\
p=0.0000\end{array}$ & $\begin{array}{l}z=11.349 \\
p=0.0000\end{array}$ & $\begin{array}{l}z=5.4071 \\
p=0.0000\end{array}$ \\
\hline $\begin{array}{l}\text { Teste de Breusch-Godfrey - } \\
\text { Autocorrelação }\end{array}$ & $\begin{array}{c}\text { Chi-squared }=59.382 \\
p=0.0000\end{array}$ & $\begin{array}{c}\text { Chi-squared }=54.446 \\
p=0.0000\end{array}$ & $\begin{array}{c}\text { Chi-squared }=36.879 \\
p=0.0000\end{array}$ & $\begin{array}{c}\text { Chi-squared }=37.426 \\
p=0.0000\end{array}$ & $\begin{array}{c}\text { Chi-squared }=39.61 \\
\qquad p=0.0000\end{array}$ & $\begin{array}{c}\text { Chi-squared }=49.637 \\
p=0.0000\end{array}$ & $\begin{array}{c}\text { Chi-squared }=40.348 \\
p=0.0000\end{array}$ \\
\hline $\begin{array}{l}\text { Teste de Wooldridge - } \\
\text { Autocorrelação em modelos } \\
\text { com efeitos fixos }\end{array}$ & $\begin{array}{c}\text { Chi-squared }=35.139 \\
p=0.0000\end{array}$ & $\begin{array}{c}\text { Chi-squared }=32.327 \\
p=0.0000\end{array}$ & $\begin{array}{l}\text { Chi-squared }=29.07 \\
\qquad p=0.0000\end{array}$ & $\begin{array}{c}\text { Chi-squared }=24.426 \\
p=0.0000\end{array}$ & $\begin{array}{c}\text { Chi-squared }=28.457 \\
p=0.0000\end{array}$ & $\begin{array}{c}\text { Chi-squared }=30.031 \\
p=0.0000\end{array}$ & $\begin{array}{c}\text { Chi-squared }=28.232 \\
p=0.0000\end{array}$ \\
\hline $\begin{array}{l}\text { Teste de Breusch - Pagan - } \\
\text { Heterocedasticidade }\end{array}$ & $\begin{array}{l}\mathrm{BP}=145.47 \\
\mathrm{p}=0.0000\end{array}$ & $\begin{array}{l}B P=169.91 \\
p=0.0000\end{array}$ & $\begin{aligned} B P & =157.87 \\
p & =0.0000\end{aligned}$ & $\begin{array}{l}\mathrm{BP}=165.75 \\
\mathrm{p}=0.0000\end{array}$ & $\begin{array}{c}B P=153.69 \\
p=0.0000\end{array}$ & $\begin{array}{l}B P=168.06 \\
p=0.0000\end{array}$ & $\begin{array}{c}\mathrm{BP}=168.81 \\
\mathrm{p}=0.0000\end{array}$ \\
\hline
\end{tabular}

Quadro 1 - Testes de especificação e diagnóstico dos resíduos para as regressões observadas no Quadro 2

Fonte: Elaborado pelo autor (2016) 
variáveis e também que os anos de estudo ainda explicam melhor o crescimento nos estados brasileiros que o resultado do Saeb para a quarta série. Um dos motivos que se apresentam de maneira mais clara é o fato de que os alunos da quarta série ainda irão demorar para ingressar no mercado de trabalho, e os efeitos de produtividade sobre o PIB podem ainda não estar presentes na amostra. $\mathrm{O}$ resultado do $R^{2}$ ajustado também cresceu ao incluir os anos de estudo na estimação, indo de 0.13 para 0.26 . O teste $\mathrm{F}$ por sua vez mostra a significância conjunta dos coeficientes, atestando que não são todos iguais a zero. Uma das características da estimação por efeitos fixos é a impossibilidade de observar coeficientes de variáveis fixas no tempo, que desaparecem do modelo com a transformação realizada na equação utilizada para a estimação. Dessa forma, algumas características regionais não podem ser isoladas, ficando presentes na parte não idiossincrática dos erros, associadas a outras características individuais.

A terceira e quarta especificações do modelo diferem da primeira e da segunda ao substituir os resultados no Saeb da quarta série pelos do terceiro ano do segundo grau nas regressões. Em relação a especificação (3), o coeficiente do resultado do terceiro ano apresentou resultado positivo, significante e próximo do coeficiente do resultado da quarta série da especificação (1). Foi possível constatar uma diferença maior ao incluir os anos de estudo para pessoas com 25 anos ou mais na regressão, enquanto o resultado para a quarta série perdeu a significância estatística, o resultado para o terceiro ano manteve num intervalo de $95 \%$ de confiança, o que indica que a proximidade dos alunos do terceiro ano de ingressarem no mercado de trabalho pode fazer com que a amostra capture diferenças na produtividade observadas pelas habilidades cognitivas. Quanto aos anos de estudo, apresentaram resultado similar ao obtido nas duas primeiras regressões.

A quinta regressão exclui da análise os anos de estudo e coloca os resultados tanto da quarta série quanto do terceiro na estimação. Nesse caso, os parâmetros apresentam sinal positivo e próximo das estimações em que os resultados dos testes foram utilizados individualmente, e significância estatística num nível crítico de $1 \%$. Ao fazer uma estimação apenas com os anos de estudo, na regressão (6) do Quadro 2, o coeficiente, da mesma forma, apresentou significância estatística. Por fim, a última estimação apresenta as três variáveis simultaneamente, sendo elas os anos de estudo médios para pessoas com 25 anos ou mais e os resultados do Saeb para a quarta série e para o terceiro ano do ensino médio. Nesse caso, para os resultados da quarta série não houve significância estatística, enquanto que para os resultados do terceiro ano e para os anos de estudo médios houve. Isso reforça o que foi observado nas regressões anteriores, que os resultados do terceiro 
ano explicam melhor o crescimento nos estados brasileiros que os resultados da quarta série, para a amostra selecionada. Também demonstra, que para o período, os anos de estudo médios são significativos nos diferenciais de PIB. Entre as sete regressões, a última foi a que apresentou o maior $R^{2}$ ajustado, sendo ele igual a 0.31 .

\begin{tabular}{|c|c|c|c|c|c|c|c|}
\hline Variáveis & (1) & (2) & (3) & (4) & (5) & (6) & (7) \\
\hline \multirow{2}{*}{$\begin{array}{l}\text { log(indústria } \\
\text { transformação) }\end{array}$} & 0.0183 & 0.0286 & 0.0257 & 0.0359 & 0.0300 & 0.0287 & 0.0359 \\
\hline & 0.0317 & 0.334 & 0.0378 & 0.0178 & 0.0355 & 0.0344 & 0.0368 \\
\hline \multirow{2}{*}{$\begin{array}{l}\text { log(matemática 4ạ } \\
\text { série) }\end{array}$} & $0.2647^{* * *}$ & 0.0675 & & & $0.2308^{* * *}$ & & 0.0735 \\
\hline & 0.0623 & 0.608 & & & 0.0537 & & 0.0555 \\
\hline \multirow{2}{*}{$\begin{array}{l}\text { log(matemática 3o } \\
\text { ano) }\end{array}$} & & & $0.2462 * * *$ & $0.1633^{* *}$ & $0.2143 * * *$ & & $0.1648 * * *$ \\
\hline & & & 0.0659 & 0.0582 & 0.0609 & & 0.0430 \\
\hline \multirow{2}{*}{$\log ($ anos de estudo) } & & $0.3298 * * *$ & & $0.3219 * * *$ & & $0.3713^{* * *}$ & $0.2763 * * *$ \\
\hline & & 0.0745 & & 0.0691 & & 0.0742 & 0.0579 \\
\hline Observações & 162 & 162 & 162 & 162 & 162 & 162 & 162 \\
\hline $\begin{array}{l}\text { Soma dos quadrados } \\
\text { dos resíduos }\end{array}$ & 0.4643 & 0.3755 & 0.4653 & 0.3422 & 0.39633 & 0.3794 & 0.3377 \\
\hline $\mathrm{R}^{2}$ & 0.1679 & 0.3269 & 0.1661 & 0.3866 & 0.2897 & 0.3200 & 0.3947 \\
\hline $\mathrm{R}^{2}$ ajustado & 0.1378 & 0.2663 & 0.1363 & 0.315 & 0.236 & 0.2627 & 0.3192 \\
\hline Estatística F & 13.4201 & 21.3691 & 13.248 & 27.7419 & 17.9480 & 31.3009 & 21.3638 \\
\hline $\mathrm{p}$ valor (Estatística F) & 0.0000 & 0.0000 & 0.0000 & 0.0000 & 0.0000 & 0.0000 & 0.0000 \\
\hline
\end{tabular}

Quadro 2 - Resultado das regressões

Fonte: Elaborado pelo autor

A utilização de inferência robusta, a partir do método proposto por Arellano (1987), é justificada pela presença de correlação entre os elementos da cross-section, correlação serial e heterocedasticidade. O Quadro 1 apresenta os testes utilizados. Para a correlação entre os elementos da cross-section foram utilizados o teste de Breusch- Pagan e o teste de Pesaran, e ambos apontaram a existência de correlação. Os testes de BreuschGodfrey e Wooldridge, com ambos apontando a existência de autocorrelação. Para a heterocedasticidade utilizou-se o teste de Breusch-Pagan e a hipótese nula, de homocedasticidade, foi rejeitada. Assim sendo, a não utilização de inferência robusta poderia resultar em interpretações equivocadas quanto à significância dos coeficientes encontrados. 


\section{CONCLUSÃO}

O capital humano é elemento chave para o crescimento econômico em países e regiões, ao impulsionar a produtividade do trabalho, insumo básico em qualquer atividade produtiva. No entanto, como não é diretamente observável, ao estimar o impacto do estoque ou fluxo de capital humano é necessário escolher proxies, em geral associadas a escolaridade. Como demonstra Cunha et al (2006), ao utilizar apenas as habilidades adquiridas a nível escolar, negligencia-se aspectos referentes as habilidades cognitivas e não-cognitivas, elementos básicos para a formação do capital humano, oriundas das famílias, da pré-escola e das próprias habilidades natas. A utilização de testes de conhecimentos que captam as habilidades cognitivas dos indivíduos se apresentam como recurso para a inserção do capital humano em modelos empíricos, desde os trabalhos de Hanushek e Kimko (2000), ao incorporar muitos dos elementos citados, além de destacar a diferença de retorno na escolaridade em diferentes locais. Além disso, como argumenta Cunha et al (2006), as habilidades cognitivas e não cognitivas possuem inter-relação, de forma que uma potencializa a outra, ou seja, ao observar o desempenho em termos de habilidades cognitivas parte dela deriva das habilidades não cognitivas.

No presente trabalho foi observado o crescimento do PIB per capita em relação ao desempenho dos alunos nas provas do Saeb, para a quarta série e terceiro ano do ensino médio, e anos médios de escolaridade nos estados brasileiros. Além disso, foi utilizada uma proxy para o capital físico per capita, sendo ela o PIB da indústria de transformação per capita. Essa variável, no entanto, mostrou-se não significativa em nenhuma das sete especificações estimadas, o que indica que o PIB da indústria de transformação não é uma boa proxy, para o capital físico nos estados brasileiros. Observando os desempenhos na prova do Saeb, bem como os anos médios de escolaridade, foi possível constatar que os melhores desempenhos se encontram nos estados do Sul e Sudeste acrescidos do Distrito Federal. $\mathrm{O}$ fato dos melhores desempenhos em média encontrarem-se nas mesmas áreas indica o efeito auto-produtivo das habilidades, além da complementaridade dinâmica, ou seja, alunos com melhor desempenho na quarta série tendem a apresentar também melhor desempenho no terceiro ano, já que o acumulo de capital humano é um processo cumulativo. Ao possuir etapas mais fracas em termos de aquisição de habilidades, o indivíduo também fica sujeito a perder períodos críticos ou sensíveis na formação do capital humano, o que significa a impossibilidade, ou maior custo futuro para adquirir determinadas habilidades, como o aprendizado de uma nova língua, por exemplo, que 
como destacam Cunha e Heckman (2007), apenas na infância é possível de ser aplicada sem sotaque.

Com relação aos resultados da estimação, para a prova de matemática do Saeb aplicada a quarta série, o resultado foi positivo e com significância estatística apenas quando estimada de maneira isolada ou acrescida do resultado do terceiro ano do ensino médio. Quando estimada em conjunto com os anos de estudo médios a os coeficientes perdem a significância estatística. Uma das explicações, como apresenta Haushek e Woessman (2012a), é que ao utilizar indivíduos mais jovens para testar os retornos da produtividade do trabalhador é possível que ao longo da amostra eles ainda não tenham se inserido no mercado de trabalho, o que dificulta a análise. Já o desempenho dos coeficientes associados ao resultado do terceiro ano do ensino médio foram significativos e positivos em todas as estimações, o que aponta que a variável explica bem o crescimento econômico nos estados brasileiros. Ao incluir os anos de estudo médios para pessoas com 25 anos ou mais, buscou-se comparar os testes cognitivos com uma variável associada à escolaridade. O que é possível constatar é que os anos médios de estudo possuem efeitos positivos e bom desempenho estatístico quando utilizadas para estimar o crescimento dos estados brasileiros, desempenho inclusive melhor em alguns aspectos, como o $\mathrm{R}^{2}$ quando as variáveis são utilizadas isoladamente, do que as variáveis associadas às habilidades cognitivas.

È possível concluir que as habilidades cognitivas são bom elemento explicativo para o crescimento econômico para os estados brasileiros, principalmente se utilizadas para indivíduos com idades maiores, ou mais próximos de entrar no mercado de trabalho. Também é possível observar, via anos de estudo para pessoas com 25 anos ou mais, que ao associar variáveis relacionadas ao temo de escolaridade a estimação pode ser melhorada, embora algumas variáveis possam perder significância estatística.

\section{REFERÊNCIAS}

ARROW, K. The economic implications of learning by doing. Review of Economic Studies v. 29, p.155-173, 1962.

BARNETT, W. S. Benefit-Cost Analysis of Preschool Education. http://nieer.org/ resources/files/BarnettBenefits.ppt, 2004.

BARRO, R. J. Economic Growth in a Cross Section of Countries. Quarterly Journal of Economics, v.106, n.2, pp. 407-43, 1991. 
BARRO, R. J.; LEE, J-W. International Comparisons of Educational Attainment. Journal of Monetary Economics, v.32, n.3, pp. 363-94, 1993.

BECKER, G. Human Capital: A Theoretical and Empirical Analysis, with Special Reference to Education. Chicago: University of Chicago Press, 1964.

BECKER, G.; MURPHY, K.; TAMURA, R. Human capital, fertility, and economic growth. Journal of Political Economy, v.98, n.5, pp.12-37, 1990.

BECKER, G.; TOMES, N. Human Capital and the Rise and Fall of Families. Journal of Labor Economics, v.4 n.3, pp. 1-39, 1986.

BENHABIB, J.; SPIEGEL, M. M. The Role of Human Capital in Economic Development: Evidence from Aggregate Cross- Country Data. Journal of Monetary Economics, v.34, n.2, pp. 143-174, 1994.

BILS, M.; KLENOW, P. J. Does Schooling Cause Growth? American Economic Review, v.90, n.5, pp. 1160-83, 2000.

BLAU, D.; CURRIE J. Preschool, Daycare, and Afterschool Care: Who's Minding the Kids? In HANUSHEK, E, A.; WELCH, F (Eds). Handbook of the Economics of Education, Vol 2. Amsterdam:North-Holland, pp.1163-1278, 2006.

CARNEIRO, P.; HECKMAN, J. The Evidence on Credit Constraints in Post- Secondary Schooling. Economic Journal, v.112 n.482, pp. 705-734, 2002.

CARNEIRO, P.; HECKMAN, J. Human Capital Policy. In Inequality in America: What Role for Human Capital Policies?, ed. James J. Heckman, Alan B. Krueger and Benjamin M. Friedman. Cambridge, MA: MIT Press, 2003.

CUNHA, F.; HECKMAN, J. The technology of skill formation. American Economic Review, v.97 n.2, 31- 47, 2007.

CUNHA, F.; HECKMAN, J.; LOCHNER, L.; MASTEROV, D. Interpreting the Evidence on Life Cycle Skill Formation. In HANUSHEK, E, A.; WELCH, F (Eds). Handbook of the Economics of Education. Amsterdam: North-Holland : Elsevier, pp.697-812, 2006.

CURRIE, J.; THOMAS, D. Does Head Start Make a Difference? American Economic Review, v.85, n.3, pp.341-364, 1995

HANUSHEK, E. A.; KIMKO, D. Schooling, Labor Force Quality, and the Growth of Nations. American Economic Review, v.90, n.5, pp.1184-1208, 2000.

HANUSHEK, E. A.; WOESSMANN, L. The Role of Cognitive Skills in Economic Development. Journal of Economic Literature, v.46, n.3, pp.607-668, 2008.

HANUSHEK, E. A.; WOESSMANN, L. The economics of international differences in educational achievement. In HANUSHEK, E. A.; MACHIN, S.; WOESSMAN L. (Eds.), 
Handbook of the Economics of Education, Vol. 3. Amsterdam: North Holland, pp.89$200,2011$.

HANUSHEK, E. A.; WOESSMANN, L. Do better schools lead to more growth? Cognitive skills, economic outcomes, and causation. Journal of Economic Growth, v.17, n.4, pp.267-321, 2012a.

HANUSHEK, E A.; WOESSMANN, L. Schooling, educational achievement, and the Latin American growth puzzle. Journal of Development Economics, v.99, n.2, pp.497$512,2012 b$.

HECKMAN, J. Lessons from The Bell Curve. Journal of Political Economy, v.103, n.5 pp.1091-1120, 1995.

HECKMAN, J.; STIXRUD J.; URZUA. S. The Effects of Cognitive and Noncognitive Abilities on Labor Market Outcomes and Social Behavior. Journal of Labor Economics, v.24, n.3, pp.411-82, 2006.

KNUDSEN, E.I. Sensitive periods in the development of the brain and behavior. Journal of Cognitive Neuroscience, v.16, n.1, pp.1412-1425, 2004.

LEE, J-W.; BARRO, R. J. Schooling Quality in a Cross Section of Countries. National Bureau of Economic Research (Cambridge, MA) Working Paper No. 6198, September 1997.

LEVINE, R.; RENELT, D. A Sensitivity Analysis of Cross-Country Growth Regressions. American Economic Review, v.82, n.4, pp. 942-63, 1992.

LUCAS, R. E. On the Mechanics of Economic Development. Journal of Monetary Economics, v.22, n.1, pp.3-42, 1988.

MANKIW, N. G.; ROMER, D.; WEIL, D. A Contribution to the Empirics of Economic Growth. Quarterly Journal of Economics, v.107, n.2, pp.407-37, 1992.

NELSON, R. R.; PHELPS, E. Investment in Humans, Technology Diffusion, and Economic Growth. American Economic Review, v.56, n.2, pp.69-75, 1966.

ROMER, P. Increasing returns and long-run growth. Journal of Political Economy, v.94, n.5, pp.1002-1037, 1986.

ROMER, P. Growth based on increasing returns due to specialization. American Economic Review, v.77, n.1, pp.56-62, 1987

ROMER, P. Endogenous Technological Change. Journal of Political Economy, v.98 n.5, pp.71-102, 1990a.

ROMER, P. Human Capital and Growth: Theory and Evidence. Carnegie-Rochester Conference Series on Public Policy, v.32, pp.251-86, 1990 b. 
RUTTER, M. Genes and Behavior: Nature-Nurture Interplay Explained. Oxford: Blackwell Publishing, 2006.

SHONKOFF, J.; PHILLIPS, D. From Neurons to Neighborhoods: The Science of Early Childhood Development. Washington, DC: National Academy Press, 2000.

UZAWA, H. Optimum technical change in an aggregative model of economic growth. International Economic Review, v. 6, n.1, pp. 18-31, 1965. 
